\title{
PLATAFORMA GIROSCÓPICA REALIZADA MEDIANTE IMPRESIÓN 3D PARA EL CONTROL DE ACTITUD Y ORIENTACIÓN DE UAVS MULTI-ROTOR
}

\author{
S. Nájera, J. Rico-Azagra, C. Elvira, M. Gil-Martínez \\ Grupo de Ingeniería de Control, Departamento de Ingeniería Eléctrica, Universidad de la Rioja, Logroño \\ \{silvano.najera, javier.rico, carlos.elvira, montse.gil\}@unirioja.es
}

\begin{abstract}
Resumen
El presente trabajo muestra el desarrollo y construcción de una plataforma de tipo giróscopo de 3 ejes, enfocada para el análisis, síntesis y prueba de algoritmos de control de UAVs de tipo multi-rotor con una distancia entre ejes máxima de $25 \mathrm{~cm}$. La estructura orbital se compone de piezas impresas en PET (polietileno), y diversos elementos estructurales $y$ constructivos que permiten, entre otras funcionalidades: la alimentación externa del UAV, la transmisión de señales mediante comunicación USB, y la obtención de ángulos de giro absolutos de los diferentes ejes que lo forman. Este sistema pretende ser usado en docencia e investigación, debido a su robustez, replicabilidad, relativo bajo coste, y seguridad en su empleo. La plataforma permitirá adquirir conocimientos, y validar en laboratorio técnicas de modelado experimental y estrategias de control de actitud y orientación de UAVs multi-rotor.
\end{abstract}

Palabras clave: Vehículo aéreo no tripulado (UAV), equipo didáctico de bajo coste, maqueta giroscópica/orbital, banco de pruebas

\section{INTRODUCCIÓN}

Los vehículos aéreos no tripulados (UAVs) de tipo multi-rotor han experimentado un amplio desarrollo en los últimos años, debido principalmente a la despiadada competencia del mercado de consumo, a la proliferación de normativas que rigen el uso de drones civiles, y a una gran efervescencia de emprendedores en el sector. Cierto es que los UAVs de tipo multi-rotor tienen un gran potencial, tanto en aplicaciones civiles como militares. Algunas de las muchas aplicaciones de estos UAVs son la inspección de infraestructuras [14], la agricultura de precisión [2], o la reconstrucción de terrenos en tres dimensiones [15].

Además, la difusión de estos usos en los medios de comunicación, ha propiciado la popularización de este tipo de aeronaves entre la sociedad y los aficionados al radiocontrol. La creciente demanda de material ha fomentado el afloramiento de numerosas empresas dentro del sector, y con éstas el aumento de la competencia. La reducción de los costes de adquisición de equipos ha provocado que los UAV multi-rotor sean cada vez más populares y accesibles [11].

De la misma forma, las tesis doctorales, los proyectos fin de carrera, y los trabajos fin de grado-máster sobre esta temática han aumentado en los últimos años [4]. También han aflorado concursos de Ingeniería de Control [1] en el marco de las Jornadas de Automática que han versado sobre esta temática.

Desde el punto de vista de la ingeniería, el gobierno de un UAV comprende un conjunto muy amplio de disciplinas, de entre las que destacan: la ingeniería de control, los sistemas de estimación, las comunicaciones, el procesado de señal, o los sistemas embebidos. Además, los UAV se pueden emplear tanto en docencia como en investigación para comprobar los conceptos teóricos adquiridos en las diferentes disciplinas de la ingeniería. Al mismo tiempo, es una temática atractiva para los estudiantes, que permite motivarlos y mejorar el aprendizaje. Sin embargo, la complejidad de su configuración, la necesidad de espacios cerrados suficientemente grandes para su vuelo, su fragilidad frente a impactos, o el riesgo para los usuarios son algunos de los inconvenientes que presentan estos sistemas para su uso en docencia.

En respuesta a estos inconvenientes, algunos trabajos han presentado algunas soluciones parciales a estos problemas [16], y también existen en el mercado plataformas didácticas [6][9][12], pero su coste económico es muy elevado.

Este trabajo presenta el desarrollo de una plataforma didáctica de bajo coste, realizada parcialmente mediante impresión 3D. La plataforma presentada se muestra como una alternativa a los costosos sistemas comerciales [6][9], y que, además, es flexible a la incorporación de nuevos desarrollos. La solución presentada está compuesta por un UAV de tipo cuatri-rotor de $250 \mathrm{~mm}$ de envergadura, junto con una estructura mecánica que permite su rotación en los tres ángulos de Euler sin presentar movimiento 
lineal. La configuración y programación del sistema UAV se desarrolla íntegramente en Matlab-Simulink [7].

El uso de la plataforma está dirigido a la docencia de asignaturas relacionadas con la ingeniería de control, en grados y másteres profesionales $\mathrm{o}$ de investigación. Los estudiantes pueden enfrentarse a problemas de modelado y control realistas y con diferentes grados de dificultad.

\section{DISEÑO DE LA PLATAFORMA}

Con anterioridad al desarrollo de esta plataforma, se han realizado prototipos [13] utilizando diferentes materiales y procedimientos constructivos. Los problemas surgidos en dichas experiencias han contribuido a las mejoras introducidas en la nueva plataforma. Algunos de estos problemas son:

- El agotamiento de la batería y, por tanto, la necesaria supervisión e interrupción de los experimentos para el cambio de ésta.

- La pérdida de datos mediante comunicación por telemetría dependiendo del entorno radioeléctrico.

- Los materiales usados pueden generar grandes inercias debido a su masa y su distancia respecto al centro de masas.

- La dificultad para alinear el centro de gravedad del dispositivo multi-rotor con el centro geométrico de la plataforma orbital.

- La dificultad para el correcto equilibrado de la plataforma completa en los 3 ejes, provocado por asimetrías en su construcción.

- Una baja seguridad en el empleo de forma remota sin supervisión.

- Los problemas debidos al ruido eléctrico acoplado en la transmisión de señales de comunicación.

- La compleja reproductibilidad de la plataforma y los elevados costes.

- La dificultad para cablear la plataforma y para pasar convenientemente señales $\mathrm{y}$ alimentación desde el exterior (estructura de soporte) al interior (donde se aloja el UAV).

En la Figura 1 se pueden apreciar otros prototipos previos al diseño de esta plataforma. Respecto a la plataforma software necesaria para el funcionamiento, se parte del firmware y software en Matlab/Simulink desarrollado previamente según [13].

A continuación, se describe el desarrollo y configuración de la plataforma, diseñada para evitar y minimizar los problemas destacados anteriormente.

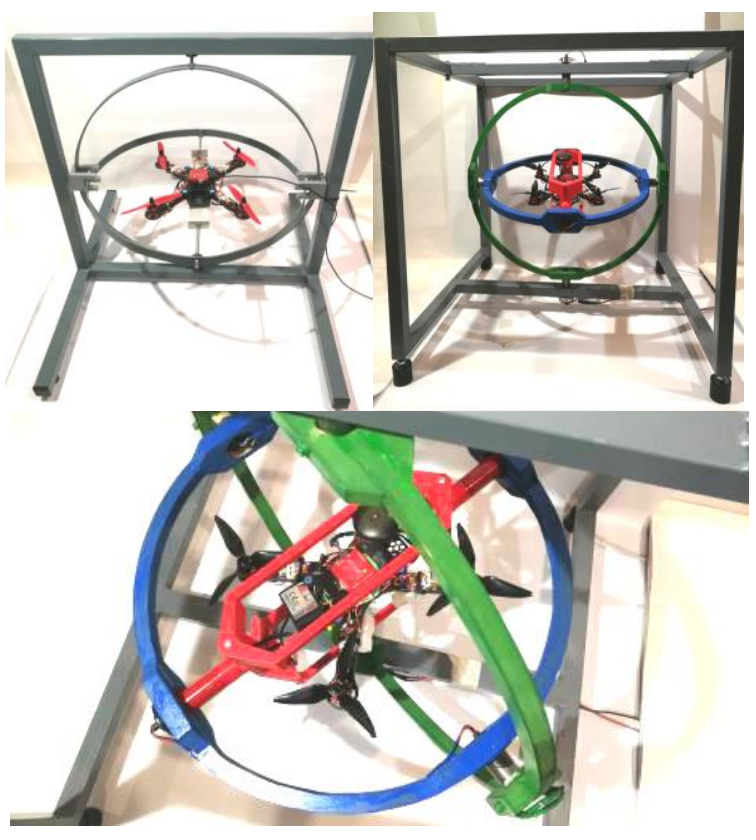

Figura 1: Prototipos previos de la plataforma desarrollada

\subsection{Estructura mecánica}

La plataforma de tipo giroscópico de 3 DOF se integra en un bastidor conformado por tubo de acero $30 \mathrm{~mm} \times 30 \mathrm{~mm} \times 1 \mathrm{~mm}$, soldado formando una estructura cúbica de $720 \mathrm{~mm}$ de lado. La estructura se dispone de esta forma según la Figura 2, para permitir el cierre completo mediante una malla de seguridad que evite el posible contacto de personas con las unidades de giro. Los tubos delanteros y traseros inferiores se eliminan para permitir un mejor acceso al dispositivo. El bastidor se completa con unas patas de tipo silent-block, regulables en altura para permitir un correcto nivelado y para reducir las vibraciones producidas. La plataforma orbital se monta sobre dos tubos aleteados centrales fijados mediante tornillería a la parte inferior y superior de la estructura.

La estructura orbital está formada por 2 aros concéntricos (de $58 \mathrm{~cm}$ y $47 \mathrm{~cm}$ de diámetro exterior, respectivamente), que giran sobre ejes orientados formando $90^{\circ}$ entre sí, y una plataforma a lo largo de un tercer eje (orientado a $90^{\circ}$ con respecto al eje del aro interior), que sustenta al UAV.

Los aros han sido diseñados y fabricados mediante impresión 3D en PET, siendo este un material que tiene buenas prestaciones frente a esfuerzos y baja biodegradabilidad frente a agentes externos como rayos UV. Tras varias pruebas de esfuerzo, el relleno empleado ha sido del $20 \%$, buscando un equilibrio entre una menor densidad que reduzca los momentos 
de inercia, y una mayor resistencia frente a los esfuerzos a los que estará sometido el material. Durante las pruebas de impresión, también se han determinado otros factores como la altura de capa $(0.2 \mathrm{~mm})$ y las velocidades de impresión $(30 \mathrm{~mm} / \mathrm{s})$, para que las piezas impresas tengan suficiente calidad como para que no se vean comprometidas ante los esfuerzos habituales a los que serán sometidas durante los ensayos. Para su fabricación $\mathrm{y}$ reproducibilidad en impresoras $3 \mathrm{D}$ convencionales, los aros se han descompuesto en tramos más pequeños, usándose la técnica de machihembrado (Figura 3). Se utilizan 6 tramos para el anillo interior y 8 para el exterior. Las piezas impresas disponen de agujeros en su interior para el paso de los cables, y se ensamblan mediante imprimación de agarre específica para PET y adhesivo de montaje en base cianocrilato. Las piezas diseñadas en $3 \mathrm{D}$ están disponibles en el repositorio http://www.thingiverse.com, bajo licencia Creative Commons, en régimen de Atribución - No Comercial para su descarga y reproducción [10].

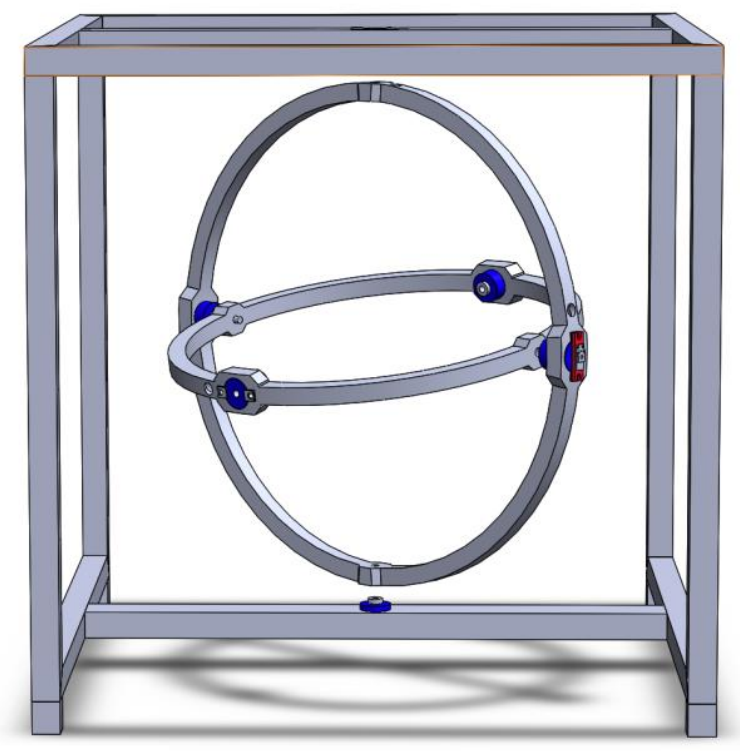

Figura 2: Modelo 3D de la plataforma giroscópica.

La plataforma interior se ha diseñado, según la Figura 4, en chapa de aluminio de $1.5 \mathrm{~mm}$ de espesor, plegada y cortada por láser, con agujeros rasgados tipo guía en sus extremos para el equilibrado del centro de masas del dispositivo UAV respecto al centro geométrico de la plataforma giroscópica.

Entre cada uno de estos elementos móviles, y entre el bastidor y el anillo exterior, se colocan anillos eléctricos rozantes de forma simétrica para la transmisión de señales y comunicación USB, por un lado, y la alimentación del UAV por otro, tal como se aprecia en la Figura 2. Los anillos rozantes seleccionados son de reducidas dimensiones y $50 \mathrm{~g}$ de peso, y poseen 12 circuitos de hasta $5^{\mathrm{a}}$, cada uno. Esto permite la transmisión de señales $\mathrm{y} / \mathrm{o}$ alimentación hasta 30A. Estos anillos rozantes o sliprings disponen de rodamientos integrados que son capaces de soportar el peso y las fuerzas provocados en el conjunto, y la unión entre los anillos rozantes y los aros y plataforma interior se realiza mediante tornillería basada en tuercas embutidas y varilla semi-roscada de $8 \mathrm{~mm}$.
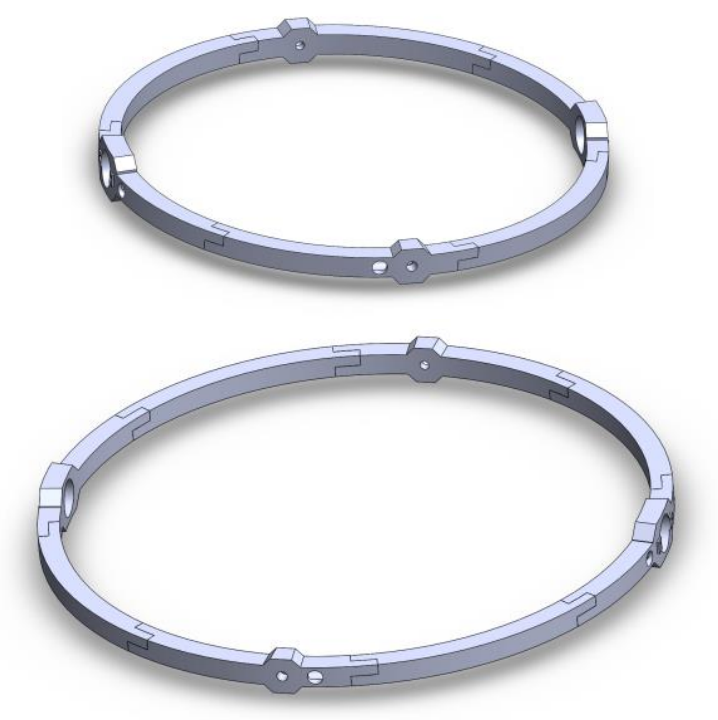

Figura 3: Descomposición de piezas de los anillos para la impresión 3D; arriba anillo interior, abajo anillo exterior.

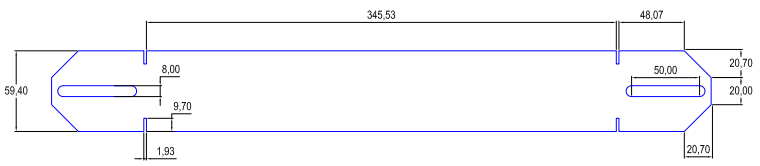

Figura 4: Desarrollo de plataforma interior para soporte de UAV

Los anillos rozantes evitan el uso de batería en el UAV y los problemas que ello conlleva, y permiten prescindir de comunicación por telemetría (programación de firmware y comunicación en tiempo real), al mismo tiempo que reducen el riesgo de fallos por ruido eléctrico acoplado al permitir el paso de señales y alimentación de elementos de potencia de forma separada. Además, debido a la minimización del peso de los materiales, se reduce la inercia del sistema. La recreación mediante impresión 3D proporciona exactitud en la distribución del peso, y el empleo de componentes distribuidos simétricamente hace que la plataforma se encuentre equilibrada desde el inicio de su construcción. Además, todo el diseño se ha ejecutado pensando en la reducción de costes, la simplicidad en el montaje y la reproducibilidad de éste. De esta 
forma, la estructura diseñada tiene, entre otras posibilidades, las siguientes funcionalidades:

- Posibilidad de bloqueo independiente de ejes de giro.

- Posibilidad de añadir perturbaciones mediante pesos en los diferentes aros provocando desequilibrios.

- Posibilidad de motorización mediante motores DC o BLDC para añadir perturbaciones medibles de par.

- Incorpora sensores absolutos de giro (potenciómetro o encoder), que permiten medir los giros absolutos, para contrastar con los estimados a partir de la IMU, y para conocer la orientación exacta de partida de los ensayos, entre otros posibles usos.

\subsection{Sistema eléctrico y comunicaciones}

El sistema eléctrico implementado en la plataforma, no solo es un sistema de transmisión de alimentación y señales desde donde se aloja el UAV hasta el exterior de la estructura y el PC, sino que integra una serie de sensores angulares absolutos, y la posibilidad de conexión de motores de tipo DC o BLDC para provocar perturbaciones ajustables en el sistema.

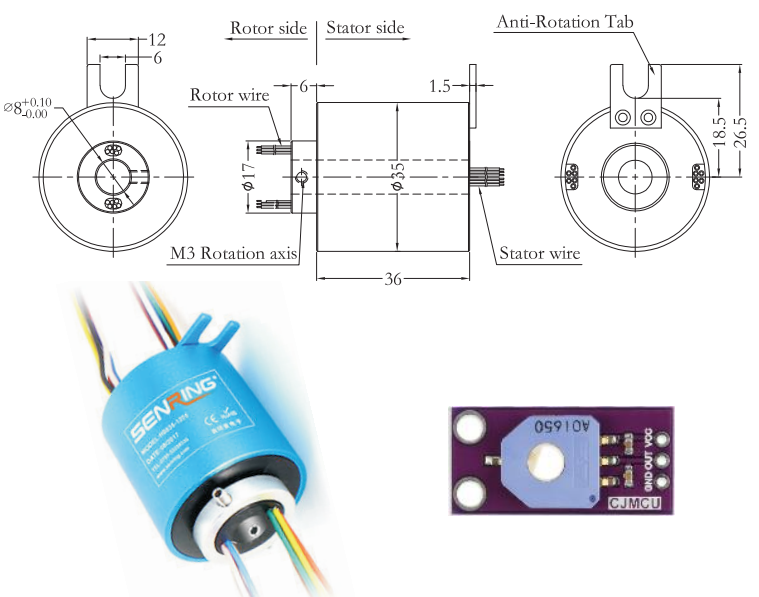

Figura 5: Slip-ring empleados (arriba y abajo izquierda) y sensor de giro absoluto (abajo derecha).

En primer lugar, el sistema eléctrico se basa en la utilización de 6 slip-rings para la conexión entre los diferentes elementos móviles, alineados simétricamente dos a dos en cada eje de giro. Los slip-rings seleccionados son los H0835-1205 del fabricante Senring (Figura 5). Poseen un diámetro interior de $8 \mathrm{~mm}$ y exterior de $35 \mathrm{~mm}$, por lo que se han fijado sobre varillas de $8 \mathrm{~mm}$ mediante prisioneros, y han sido embutidos en la estructura $\mathrm{y}$ en las piezas impresas convenientemente mediante pestañas y elementos roscados. Integran un rodamiento protegido que es capaz de soportar los esfuerzos requeridos. Estos slip-rings permiten configurar circuitos de 30A uniendo en paralelo 6 de sus circuitos de $5 \mathrm{~A}$, por lo que se ha decidido emplear un slip-ring de cada par para la alimentación del UAV, y el otro para comunicaciones y señales. De esta forma, los circuitos de alimentación y señales quedan diferenciados y separados, mientras que la simetría en volúmenes y peso se mantiene.

Por otra parte, la plataforma integra sensores de tipo potenciómetro de giro infinito, o de rotación continua, de bajo coste. Los sensores elegidos son de la marca Murata, modelo SV01A103AEA01R00 de $10 \mathrm{kohm}$, montados sobre placa (Figura 5), y se sitúan en cada uno de los ejes de rotación, permitiendo obtener el ángulo absoluto de cada uno de los 3 ejes. Poseen una linealidad del $2 \%$, garantizada en un recorrido efectivo de $320^{\circ}$.

En última instancia, se pueden montar micromotores de tipo DC o BLDC con su controlador correspondiente tipo Odrive [7], o un sistema basado en ARCP (Advanced Rapid Control Prototyping) [3]. De esta forma, se puede realizar un control de estos en par y posición, para llevar a la plataforma a un punto de origen conocido o provocar perturbaciones medibles sobre el UAV.

Por último, se ha contemplado una comunicación USB que permite tanto programar el dispositivo UAV, como monitorizar desde una estación externa, en tiempo real, el estado de sus sensores, sin tener que conectar/desconectar el mismo. Todos los elementos de los dos aros interiores están conectados a través de un bus de señales de 12 hilos desde el interior hasta el exterior a través de los slip-rings tal como se muestra en la Figura 6. El sensor del eje Z vertical, así como su micromotor opcional, solo se pueden conectar a este bus si se desconectan otros de los elementos interiores.

Por lo tanto, el sistema eléctrico diseñado proporciona las siguientes funcionalidades:

- Alimentación del UAV continua desde una fuente externa (máx. 30A), que proporciona una operación sin mantenimiento.

- Realimentación de las medidas de giro absolutas, tanto desde el UAV como desde la estación externa, permitiendo comprobar la exactitud de estimadores de giro.

- Posibilidad de crear perturbaciones de tipo par en cada uno de los ejes mediante motores.

- Posibilidad de posicionamiento automático en origen.

- Programación online y telemetría en tiempo real sin pérdida de datos. 


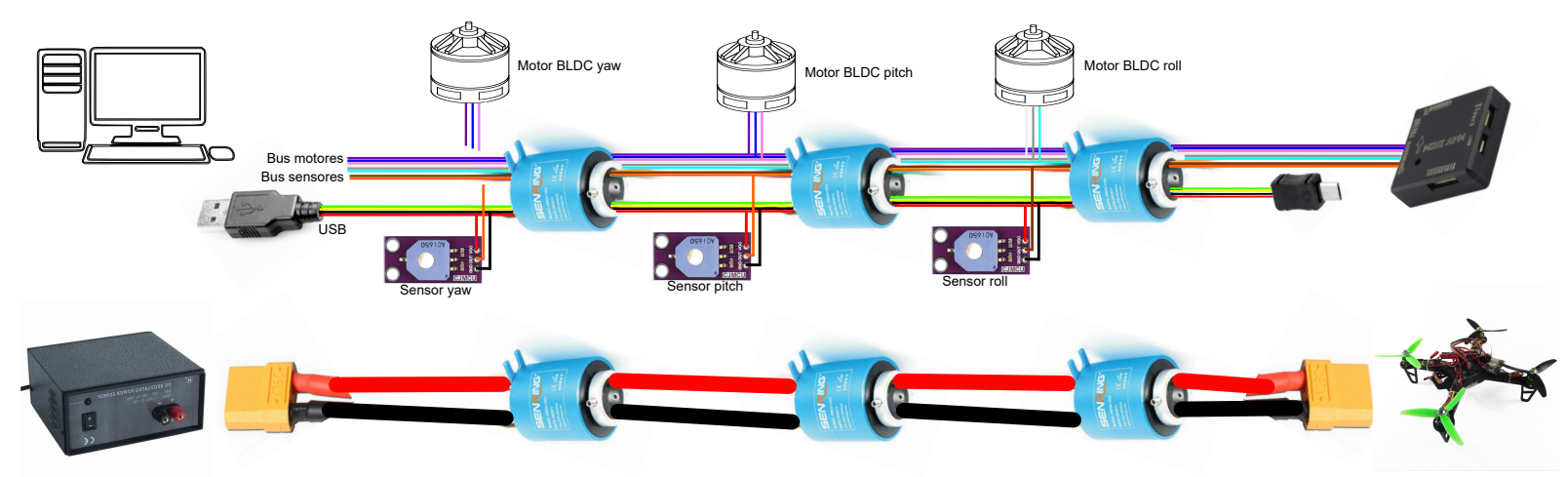

Figura 6: Esquema eléctrico de comunicaciones y señales, y elementos eléctricos de la plataforma.

\subsection{UAV tipo}

En el diseño de la plataforma se ha primado que la solución fuera segura y compacta, dado que se empleará primordialmente en un laboratorio docente. Para este propósito el tamaño comercial más adecuado de UAV es de $250 \mathrm{~mm}$ entre ejes, y es para este tamaño para el que se dimensiona la estructura tal como se explica en el Apartado 2.1.

El UAV comercial que se aloja en la plataforma actualmente diseñada consta de los siguientes elementos (Figura 7):

- $\quad$ Frame HobbyKing FPV250L, de 250mm de envergadura.

- Hélices Gemfan 5030 fabricadas en ABS.

- 4 ESC (Electronic-Speed-Control) Afro 12A opto, compatibles con baterías de 3-4s.

- 4 motores DYS BE1806 de $2300 \mathrm{kV}$.

- $\quad$ Módulo de alimentación XT60.

- Controladora de vuelo Mini APM 3.1.

- 3DR uBlox GPS con Compass kit (GPS uBlox LEA-6H and HMC5883L).

- Emisor-receptor RC de 9 canales Flysky TH9X.

El sistema de control de vuelo es accesible y configurable desde un entorno software en MatlabSimulink. Éste ha sido desarrollado para trabajar junto con las controladoras de vuelo de la familia APM (Ardu Pilot Mega) [5], que se corresponden con una modificación del sistema Arduino Mega 2560 realizada por $3 D R$ Robotics.

Puede sustituirse el UAV presentado anteriormente por cualquier unidad que presente $250 \mathrm{~mm}$ de envergadura, y que disponga de una controladora de vuelo de la familia APM 3.

Como restricción adicional, el enlace de comunicación con la emisora RC deberá disponer de al menos seis canales. Por último, hay que destacar que la comunicación serie inalámbrica es opcional, ya que se dispone de conexión USB para la monitorización del sistema durante las pruebas de vuelo. La disposición de los motores dentro del frame del multi-rotor, los sentidos de giro de éstos, y el convenio empleado para el sistema de referencia del movimiento, coinciden con los empleados en [13].

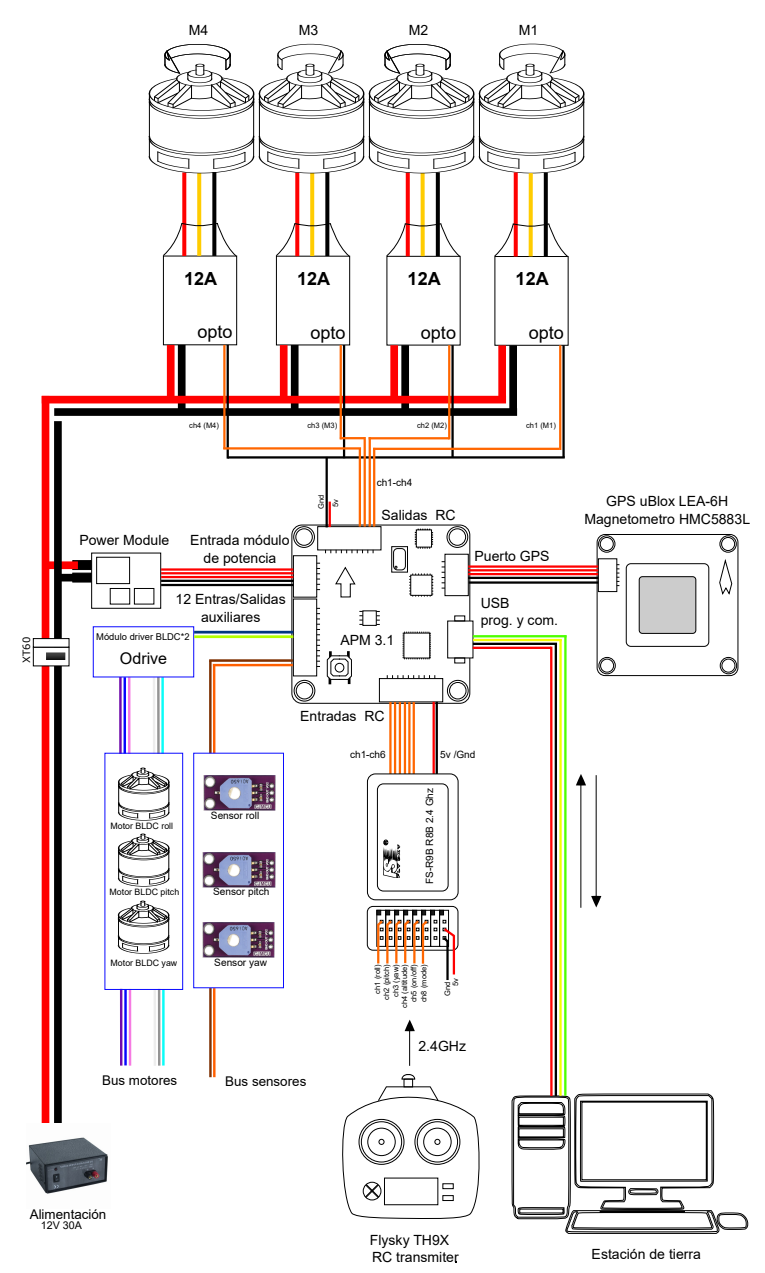

Figura 7: Esquema de componentes y conexión del UAV con la plataforma y la estación de tierra 


\section{RESULTADOS}

Tras el diseño y ejecución de la plataforma giroscópica mediante impresión $3 \mathrm{D}$, según se muestra en la Figura 8, se comprueba que todos los elementos funcionan según las características de diseño.

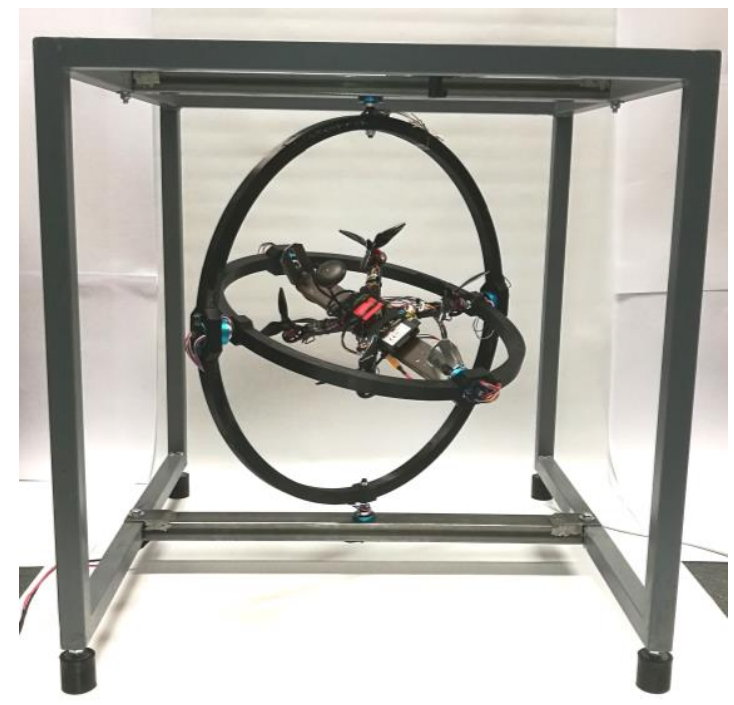

Figura 8: Plataforma giroscópica fabricada.

Tabla 1: Características de la plataforma.

\begin{tabular}{|l|l|}
\hline Característica & Valor \\
\hline Dimensiones & $72 \mathrm{~cm} \times 72 \mathrm{~cm} \times 72 \mathrm{~cm}$ \\
\hline Peso total & $14 \mathrm{~kg}$ aprox. \\
\hline Resolución giro & Según conversor A/D \\
\hline Potencia motor & Hasta $1.4 \mathrm{Nm}$ \\
\hline $\begin{array}{l}\text { Peso plataforma } \\
\text { (roll) }\end{array}$ & $54 \mathrm{~g}$ \\
\hline $\begin{array}{l}\text { Peso aro int. } \\
\text { (pitch) }\end{array}$ & $336 \mathrm{~g}$ \\
\hline $\begin{array}{l}\text { Peso aro ext. } \\
\text { (yaw) }\end{array}$ & $416 \mathrm{~g}$ \\
\hline $\begin{array}{l}\text { Ángulo roll, } \\
\text { pitch y yaw }\end{array}$ & $360^{\circ}\left(320^{\circ}\right.$ monitorizados $)$ \\
\hline Tamaño UAV & Hasta $250 \mathrm{~mm}$ de eje a eje \\
\hline Potencia & $12 \mathrm{~V} 30 \mathrm{~A}$ \\
\hline Comunicación & USB 2.0 \\
\hline
\end{tabular}

Además, la inercia del sistema es menor que en los prototipos desarrollados con anterioridad (Figura 1) al haber reducido el peso de los componentes, y ser la fricción mucho menor y más homogénea en todos los ejes. El comportamiento de la plataforma es satisfactorio.

A continuación, se muestra un presupuesto detallado del proyecto (Tabla 2) y un cuadro de tiempos (Tabla 3) para su construcción:
Tabla 2: Presupuesto de la plataforma.

\begin{tabular}{|l|c|}
\hline Elemento & Coste (€) \\
\hline Estructura & 80 \\
\hline Elementos impresos en PET & 25 \\
\hline Slip-rings (6 ud.) & 360 \\
\hline Varillas y tornillería & 18 \\
\hline Sensores angulares (3 ud.) & 6 \\
\hline Cableado y conectores & 18 \\
\hline Fuente de alimentación 30A 12V & 89 \\
\hline Mano de obra/construcción & 400 \\
\hline Total & $\mathbf{9 9 6} €$ \\
\hline
\end{tabular}

Tabla 3: Tiempos de ensamblaje.

\begin{tabular}{|l|c|}
\hline Actividad & Tiempo (h) \\
\hline Construcción estructura & $3 \mathrm{~h}$ \\
\hline Construcción elementos móviles & $8 \mathrm{~h}$ \\
\hline Cableado (slip-rings y sensores) & $6 \mathrm{~h}$ \\
\hline Conexiones y pruebas & $3 \mathrm{~h}$ \\
\hline
\end{tabular}

\section{CONCLUSIONES}

Se ha desarrollado y fabricado una plataforma giroscópica mediante impresión $3 \mathrm{D}$ en polietileno (PET), que permite girar libremente sobre 3 ejes un UAV multirotor. La plataforma puede albergar sensores de giro absoluto y micromotores BLDC. El desarrollo de esta plataforma ha dado como resultado un sistema en el que los alumnos pueden poner a prueba sin riesgos sus estrategias de control de orientación y actitud de UAV multi-rotor. Además, los sensores de giro absoluto integrados permiten obtener con suficiente precisión y bajo coste las medidas de los ángulos sin derivas, que pueden ser utilizadas para computar el error del sistema o de los estimadores empleados. La plataforma permite la rotación completa e ilimitada en torno a los 3 ejes (roll, pitch y yaw), y se pueden añadir motores para simular perturbaciones. Las piezas impresas 3D en PET aportan replicabilidad, robustez y ligereza al mismo tiempo, además de un fácil equilibrado. La plataforma diseñada permite introducir UAVs de tipo multi-rotor de tamaños hasta $250 \mathrm{~mm}$ de distancia entre ejes. La operación del UAV se puede realizar desde MATLAB, incluyendo la gestión de las señales de perturbación (tipo par, velocidad o posición) y las medidas de los sensores absolutos.

\section{Agradecimientos}

Los autores agradecen la ayuda prestada por el Gobierno de La Rioja a través del proyecto de $\mathrm{I}+\mathrm{D}$ ADER 2017-I-IDD-00035, y por la Universidad de La Rioja a través de la Ayuda a Grupos de Investigación REGI 2018/42 y de las ayudas a 
Equipos Docentes "Sistemas Robotizados" y "Redes y servidores".

\section{English summary}

\section{GYROSCOPIC PLATFORM MADE BY 3D PRINTING FOR THE CONTROL OF ATTITUDE AND ORIENTATION OF MULTIROTOR UAVS}

\begin{abstract}
This paper shows the development and construction of a 3-axis gyroscope-type platform, which allows the design and testing of control algorithms for multirotor-type UAVS. The orbital rings are made of pieces printed in PET (polyethylene); affixed slip rings provide the external powering of the UAV and the USB transmission of signals; a set of encoders measure the three rotation angles; the structure can hold up square frames with a maximum distance of $25 \mathrm{~cm}$ between rotor axes. It is remarkable its robustness, replicability, relative low cost and safety in its use. The system is intended to be used in teaching and research about experimental modelling and control engineering of multirotor UAVs.
\end{abstract}

Keywords: Unmanned aerial vehicles (UAV), lowcost didactic equipment, gyro/orbital model, test bench

\section{Referencias}

[1] Blasco X., Reynoso-Meza G., and Garcia- Nieto S. Results of the control engineering competition 2012 and convocation 2013. RIAI Revista Iberoamericana de Automática e Informática Industrial, 10(2):240-244, 2013.

[2] Candiago S., Remondino F., De Giglio M., Dubbini M., and Gattelli M. Evaluating multispectral images and vegetation indices for precision farming applications from uav images. Remote Sensing, 7(4):4026-4047, 2015.

[3] Flores A., Sabin D., Villoslada A., Blanco D. and Moreno L. Sistema Avanzado de Protipado Rápido para Control en la Educación en Ingeniería para grupos Multidisciplinares. Revista Iberoamericana de Automática e Informática industrial, 13:350-362, 2016.

[4] Garín, V.B. Diseño e implementación de una plataforma para la validación segura de algoritmos de control avanzados en vehículos aéreos no tripulados tipo quadrotors. Trabajo Final de Máster. Valencia: Universidad Politécnica de Valencia, 2017.

[5] http://www.ardupilot.com/

[6] https://eurekadynamics.com/fft-gyro/

[7] http://www.mathworks.com/matlabcentral/ fileexchange/39037-apm2-simulink-blockset

[8] https://odriverobotics.com/

[9] http://www.quanser.com/products/qball2

[10] https://www.thingiverse.com/thing:3743311

[11] Lim H., Park J., Lee D., and Kim H.J. Build your own quadrotor: Open-source projects on unmanned aerial vehicles. IEEE Robotics and Automation Magazine, 19(3):33-45, 2012.

[12] Ortega J.J. an Sigut M. Prototipo de una plataforma móvil de bajo coste para simulación de vuelo de alto realismo. Revista Iberoamericana de Automática e Informática industrial, 13:293.303, 2016.

[13] Rico-Azagra J., Gil-Martínez M., Rico R., Maisterra P. Plataforma didáctica de bajo coste para el control de actitud y orientación de UAVs multirotor. XXXVII Jornadas de automática, 2016.

[14] Sa I. and Corke P. Vertical infrastructure inspection using a Quadcopter and shared autonomy control, volume 92 of Springer Tracts in Advanced Robotics. 2014.

[15] Siebert S. and Teizer J. Mobile 3d mapping for surveying earthwork projects using an unmanned aerial vehicle (uav) system. Automation in Construction, 41:1-14, 2014.

[16] Yu Y. and Ding X. A quadrotor test bench for six degree of freedom flight. Journal of Intelligent \& Robotic Systems, 68(3):323- 338, 2012.

\section{(c) (1) (9) (2)}

Submitted for possible open access publication under the terms and conditions of the Creative Commons Attribution CC BY-NC-SA 4.0 license (https://creativecommons.org/licenses/bync-sa/4.0/deed.e 